\title{
Bistability of seizure-like bursting and silence
}

\author{
William Barnett ${ }^{\text {** }}$, Gabrielle O'Brien², Gennady S Cymbalyuk ${ }^{1}$ \\ From Twenty First Annual Computational Neuroscience Meeting: CNS*2012 \\ Decatur, GA, USA. 21-26 July 2012
}

Heart interneurons (HNs) in the third and fourth ganglia of the medicinal leech are units of the central pattern generator driving the heartbeat. When placed in bath with $\mathrm{Co}^{2+}$ and 4-aminopyridine, cells in a ganglion exhibit seizure-like activity with slow plateau oscillations [1]. In this scenario, the synaptic currents, the $\mathrm{Ca}^{2+}$ currents, and most of the $\mathrm{K}^{+}$currents are blocked. We consider this bursting activity to be a model for chemically induced seizures.

We present a novel Hodgkin-Huxley type model of the pharmacologically reduced $\mathrm{HN}$. The model contains a leak current and four voltage-gated ionic currents: a fast $\mathrm{Na}^{+}$ current, a persistent $\mathrm{Na}^{+}$current $\left(I_{\mathrm{P}}\right)$, a non-inactivating $\mathrm{K}^{+}$current $\left(\mathrm{I}_{\mathrm{K} 2}\right)$, and a hyperpolarization-activated current $\left(\mathrm{I}_{\mathrm{h}}\right)$. This model produced slow plateau-like bursting at an initial set of parameters. We systematically varied two parameters of the leak current $\left(\mathrm{g}_{\text {Leak }}\right.$ and $\left.\mathrm{E}_{\text {Leak }}\right)$ and recorded a variety of silent and oscillatory regimes.

For a given value of $E_{\text {Leak }}$, we established the range of values of $g_{\text {Leak }}$ for which the system supported bursting activity. For large values of $g_{\text {Leak }}$, the model exhibited quiescence. As $g_{\text {Leak }}$ was decreased, the equilibrium lost stability in an Andronov-Hopf bifurcation, and a saddle orbit was born. The ranges of values in $g_{\text {Leak }}$ for which the system exhibited bursting and quiescence overlapped defining a range of values in $g_{\text {Leak }}$ supporting coexistence of the two regimes. Coexistence means that an external perturbation can switch the activity from one of the stable regimes to another.

We explored bistability of bursting and silence by injecting a square pulse of current to perturb bursting activity. Pulses of current were characterized by two parameters: the amplitude of the pulse and the phase in the cycle period at which the pulse was applied. We varied the phase and amplitude through a range of values and determined whether a given pulse switched the activity from bursting to silence. We found contiguous parameter sets that satisfied this criterion.

We examined the role of each current in supporting bistability of bursting and silence. The propensity of the model to bistability of bursting and silence was defined as the range of $g_{\text {Leak }}$ for which bursting and silence coexisted. We computed this value while iteratively varying the maximal conductance of each voltage-gated current. Increasing the maximal conductance of $\mathrm{I}_{\mathrm{K} 2}$ increased the range of bistability, and there was a similar trend for $\mathrm{I}_{\mathrm{h}}$. In contrast, the range of bistability increased as the maximal conductance of $I_{P}$ decreased. We tested whether these effects were complimentary. We selected values of the maximal conductances of $I_{K 2}, I_{h}$, and $I_{P}$ that each supported the largest range of bistability found. We performed a twoparameter $\left(g_{\text {Leak }}, \mathrm{E}_{\text {Leak }}\right)$ bifurcation analysis in both the initial model and this new model. The range of bistability in the new model increased substantially over that exhibited in the initial model.

We suggest that this study could lead to the development of novel methods for controlling seizures.

\section{Acknowledgements}

This research was supported by NSF grant PHY-0750456 to GC.

\section{Author details}

${ }^{1}$ Neuroscience Institute, Georgia State University, Atlanta, GA, 30303, USA.

${ }^{2}$ Agnes Scott College, Decatur, GA, 30030, USA.

Published: 16 July 2012

\section{Reference}

1. Angstadt JD, Friesen WO: Synchronized oscillatory activity in leech neurons induced by calcium channel blockers. J Neurophysiol 1991, 66(6):1858-1873.

doi:10.1186/1471-2202-13-S1-P157

Cite this article as: Barnett et al:: Bistability of seizure-like bursting and silence. BMC Neuroscience 2012 13(Suppl 1):P157. 